\title{
Knowledge of Sexually Transmitted Infections and Barriers to Seeking Health Services among High School Adolescents in Addis Ababa, Ethiopia Amsale Cherie ${ }^{1 *}$ and Yemane Berhane
}

${ }^{1}$ School of Public Health, Addis Ababa University, Addis Ababa, Ethiopia

${ }^{2}$ Addis Continental Institute of Public Health, Addis Ababa, Ethiopia

\begin{abstract}
Background: Insufficient knowledge about Sexually Transmitted Infections (STI) and issues around accessing health services are among the major impediments to successfully prevent STIs among adolescent populations in developing countries.
\end{abstract}

Objectives: To assess knowledge of adolescents about STIs and identify the barriers to seeking reproductive health service among high school adolescents.

Methods: A cross sectional design complemented with a qualitative inquiry was the method used to collect relevant data among high school students in Addis Ababa, Ethiopia. A multistage sampling procedure was used to randomly select students across the city. The quantitative data were collected using a pre tested self administered questionnaire. Qualitative data were collected by conducting focus group discussions with purposively selected students. The analysis produced the proportion of adolescents who knew at least two symptoms of STI and associated factors. The qualitative information offers contextual understanding of the issues.

Results: In total, only 634(17.9\%) adolescents had knowledge of at least two symptoms of STIs. The reported knowledge was better among male students $[\mathrm{AOR}=1.47(95 \% \mathrm{Cl}: 1.13-2.91)]$ and students who had mothers with formal education $[\mathrm{AOR}=1.48(95 \% \mathrm{Cl}: 1.09-2.94)]$ compared to their counterparts. Overall $175(4.9 \%)$ of the students reported having symptoms of STls 12 months prior to the survey, of which $58(33.1 \%$ ) did not receive treatment. Among those reported treated, 50(42.7\%) took self medication. Major barriers for not seeking treatment for STIs were perception of unavailability, unaffordability and inaccessibility of STI services in the existing health institutions. Adolescents prefer obtaining user friendly STIs services in schools.

Conclusion: Adolescents are not aware of the symptoms of STI. Proper treatment for STI is not sought by the majority of students that reported STI symptoms. Inclusion of specific information and facts about STI in the school curriculum and providing user friendly STIs services at school can greatly reduce the risk of STIs among school adolescents.

Keywords: Adolescents; STI; Health seeking behavior; School health services; Sexual reproductive health service preferences; HIV; Unmet need

\section{Introduction}

Sexually Transmitted Infections (STIs) are major public health problems among young people globally [1-3]. Providing relevant information about STIs and availing the necessary health services is a highly desirable and effective intervention in the prevention and control of STIs [4-8]. However, the sexual and reproductive health needs of adolescents are often unmet in developing countries [1-3,9-11].

Adolescents are disproportionately affected by STIs because of their engagement in unsafe sexual practices such as multiple sexual partnerships, casual sex and inconsistent condom use. Evidences show that worldwide more than three million people are newly infected with STIs annually and $70 \%$ of the infections occur among young people [1$3,12,13]$. Demographic and Health surveys conducted in 20 countries in Sub-Saharan Africa evidenced that up to $11 \%$ of female and $16 \%$ of male adolescents reported that they had had an STI in the preceding 12 months of the study [9]. Data regarding the prevalence of STIs in Ethiopia are scarce. However, available evidences in Ethiopia reported a prevalence of STIs among antenatal clinic attendees as high as $43 \%$ [14] and among students up to $12 \%$ [15-17]. Mixed STIs have been documented among young people in Addis Ababa [18]. The prevalence of double infection with Chlamydia and gonorrhea infection among sexually active youth was $4.8 \%$ [18]. Furthermore, STIs are one of the common causes of outpatient visits [19]. These figures show the state of HIV prevention and control efforts in the country as HIV and STIs share common risk behaviors and routes of transmission. On top of that, the existence of STIs can fuel HIV acquisition and transmission [20]. Moreover, the grave health, social and psychological consequences of STIs has been indicated in previous studies [3].

The Ministry of Health of Ethiopia adopted and implemented the syndromic management of STIs guidelines from the World Health Organization in 2001. This approach recommends that STIs be managed at the primary health care unit [21]. The primary health care unit is the first level of the three-tier health system of Ethiopia [22].

Knowledge of symptoms of STIs is one of the most important prerequisites for seeking treatment timely. Furthermore, assessing factors that hinders adolescents from seeking treatment for STIs is pivotal in informing practice, policy and design appropriate interventions. However, such information among school going adolescents is meager *Corresponding author: Amsale Cherie, School of Public Health, Addis Ababa Uni-
versity, Addis Ababa, P.O. Box: 33412, Ethiopia, E-mail: amsalec2002@yahoo.com

Received April 18, 2012; Accepted May 24, 2012; Published May 28, 2012

Citation: Cherie A, Berhane Y (2012) Knowledge of Sexually Transmitted Infections and Barriers to Seeking Health Services among High School Adolescents in Addis Ababa, Ethiopia. J AIDS Clinic Res 3:153. doi:10.4172/2155-6113.1000153

Copyright: (C) 2012 Cherie A, et al. This is an open-access article distributed unde the terms of the Creative Commons Attribution License, which permits unrestricted use, distribution, and reproduction in any medium, provided the original author and source are credited. 
in Ethiopia. Therefore, the aim of this study was to assess the level of knowledge of STI symptoms and identify perceived barriers to seeking STIs services among high school adolescents.

\section{Methods}

The study utilized both quantitative and qualitative data collection methods to obtain complimentary information. The quantitative study was a cross sectional study conducted in Addis Ababa high schools. A three stage sampling techniques was used to randomly select 10 high schools based on probability proportional to the schools size first and then sections and students were selected randomly in a procedure described in detail in another paper [23].

The quantitative data were collected through a pre tested, closed ended self administered paper-pencil questionnaire. The questionnaire was first prepared in English and then translated into Amharic (the official language of Ethiopia). The questionnaire was adapted from previous studies $[24,25]$. The questionnaire comprised of questions on socio-demographic characteristics (age, sex, parental education); sexual practices; knowledge of symptoms of STIs, and barriers to seeking STIs treatment. We used the Amharic version of the questionnaire for data collection. The survey took on average 60 minutes to complete.

Knowledge of STIs was assessed based on two questions that asked: "Can you describe symptoms of STIs in women?" and "Can you describe symptoms of STIs in men?" Respondents were asked to circle the correct answer from the list of symptoms given. The list of symptoms given for both questions were: lower abdominal pain, genital discharge, burning during urination, genital ulcers, swelling around the groin, itching around/on the genitalia and I don't know. STI knowledge was assessed by scoring correct responses. Those who identified at least two correct symptoms of STIs were coded as '1' and those who did not or responded as "I do not know" as ' 0 '. The prevalence of reported symptoms of STI was calculated by asking respondents to indicate if they had STI symptoms in the last twelve months. Those who reported having STIs symptoms in the last twelve months were further asked if they sought treatment for the STIs symptoms: yes or no were the response options. Those who sought treatment were asked then when they sought treatment: the response categories given were immediately the symptom/s appeared or after some time. Those who sought treatment were also asked from where they sought treatment: private health institutions, government health institutions and pharmacies were the given choices to be circled by the respondents. Finally, those who delayed or not sought treatment for STIs symptoms were asked to offer reasons for not doing so. The quantitative data were analyzed using SPSS version 15 statistical package. Logistic regression model was used to examine the association between knowledge of symptoms of STIs and selected independent factors. First variables related to knowledge of symptoms of STIs such as age, sex, maternal education, and paternal education and ever had sex were analyzed using bivariate analyses and those variables found significant at the bivariate were further entered and analyzed using logistic regression analysis.

The qualitative component of the study collected information by conducting a series of Focus Group Discussions (FGD) with some selected students from the schools surveyed for this study. Selection of students for FGD was purposive and the main eligibility criterion: was membership in the school Anti Aids Club (AAC) and willingness to participate in the discussion. Members of AAC are believed to have rich information and free to discuss on sexual and reproductive health matters. A total of 96 students participated in four only males and four only females focus group discussions.
Focus Groups Discussions (FGDs) were chosen for this study to obtain a broad range of information with regard to the research questions. The discussion guide consisted of open ended questions related to the study objective. All discussions were conducted in a quite and conducive environment. The first author moderated the FGDs and the assistant took note. The discussions took on average two hours. Discussions were tape recorded after securing consent from the discussants.

The audio taped discussions were transcribed verbatim. The FGDS were analyzed using the qualitative content analysis method. The unit of analysis for this study was a meaningful statement, which ranged from a word to one or two complete sentences. In the first phase of analysis line by line coding of key phrases of the transcripts, in participants own words, were done. In the second phase of analysis the identified codes were grouped and subcategories formed. In the third stage of analysis thematic categories were formed through further grouping of subcategory.

Ethical clearance was secured from the Addis Ababa University School of Public Health and IRB of the College of Health Sciences. A formal letter was written to all concerned authorities and permission was secured at all levels. Adolescents between 15 and 18 years in Ethiopia are considered consenting minors and can be interviewed without parental consent. Verbal informed consent was obtained from each respondent after explaining the purpose and procedure of the study. Audio tape recording of the FGDs was made after getting informed consent from discussants.

\section{Results}

\section{Knowledge about STIs}

A total of 3543 participants responded to the self administered questionnaire; of which only 634(17.9\%) adolescents had knowledge of at least two symptoms of STIs. The low level of STI knowledge was congruent with the FGD results where discussants indicated that school adolescents and the school community in general, including AACs, are poorly informed about STIs. Discussants said: "Adolescents want to get information about the nature of STIs as well as their means of transmission and prevention. Students know more about HIV but very little about other STIs...even AACs members know very little about other STIs. Our teachers themselves don't have clear information about other STIs... we heard the names of STIs. More emphasis is given on HIV."

\begin{tabular}{|l|l|}
\hline Characteristics & Characteristics Number (\%) \\
\hline Sex & \\
\hline Male & $1789(50.50$ \\
\hline Age group & $1754(49.5)$ \\
\hline $15-16$ years & \\
\hline $17-24$ years & $796(22.5)$ \\
\hline Living arrangement & $2747(77.5)$ \\
\hline Both parents & \\
\hline Other arrangements & $1964(55.4)$ \\
\hline Paternal literacy & $1579(44.6)$ \\
\hline Illiterate and non formal & \\
\hline Formal school & $602(17.0)$ \\
\hline Maternal literacy & $2941(83.0)$ \\
\hline Illiterate and non formal & \\
\hline Formal school & $928(26.2)$ \\
\hline
\end{tabular}

Table 1: Socio-demographic Characteristics of 3543 High School Students. Addis Ababa, Ethiopia. April 2009 
Citation: Cherie A, Berhane Y (2012) Knowledge of Sexually Transmitted Infections and Barriers to Seeking Health Services among High School Adolescents in Addis Ababa, Ethiopia. J AIDS Clinic Res 3:153. doi:10.4172/2155-6113.1000153

Page 3 of 6

\section{Correlates of knowledge of symptoms of STIs}

Table 1 presents the independent correlates of knowledge of symptoms of STIs. Accordingly knowledge of symptoms of STIs were better among males [AOR=1.47 (95\%CI: 1.13-2.91)] compared to females; and among students who have mothers with some formal education $[\mathrm{AOR}=1.48$ (95\% CI: 1.09-2.94)] compared to those who have illiterate mothers.

Nearly one in 20 adolescents reported that they had symptoms of STIs in the past 12 months. Of these only $117(66.9 \%)$ sought treatment for their symptoms. Almost half of those who sought treatment delayed seeking treatment for sometime after they observed the symptoms. Of those who sought treatment, 50(42.7\%) of them treated themselves using drugs obtained from private pharmacies (Table 2).

\section{Reasons for delayed STIs treatment or for not seeking STIs treatment}

Reasons for delayed STIs treatment or for not seeking STIs treatment is depicted in Figure 1. The main reasons for delayed treatment reported by respondents were: I don't know were to go $42(70.0 \%)$, most health institutions are open during school hours $42(70.0 \%)$, I do not know what it is $41(68.3 \%)$, health professionals are not friendly $39(65.0 \%)$, I am ashamed 37(61.7\%), I didn't have money 34(56.7\%) and it was not that much serious 11(18.3\%). Reasons for not seeking treatment were: I do not know what it is $49(84.4 \%)$, most health institutions are open during school hours $49(84.40 \%)$, I don't know where to go $47(81.0 \%)$, health professionals are not friendly $47(81.0 \%)$, I am ashamed 42(72.4\%), I didn't have money 41(70.7\%), I may meet people whom I know 31(53.4\%) and it was not that much serious 18(31.0\%).

\section{Barriers to SRH service utilization}

Discussants reported several key barriers to seeking STIs treatment, in addition to low knowledge of STIs symptoms.

Availability of health services: The first barriers indicated by the participants were availability of health services. Under this major theme, three sub themes emerged: unavailability of STIs services targeting adolescents, unfriendliness of health professionals working in the existing health institutions and lack of knowledge of where to seek treatment for STIs.

\begin{tabular}{|l|l|c|c|c|}
\hline Factors & Number \% Reporting Knowledge of at least two STI symptoms & Crude Odds Ratio (95\% Cl) & Adjusted Odds Ratio (95\%CI) \\
\hline Ever had sex & Yes & $255(40.2)$ & $3.5(1.54-5.46)$ \\
\cline { 2 - 5 } & No & $379(59.8)$ & 1.00 \\
\hline Sex & Male & $351(55.4)$ & $1.64(0.98-3.42)$ \\
\cline { 2 - 5 } & Female & $283(44.6)$ & $1.33(1.09-2.64)$ \\
\hline $\begin{array}{l}\text { Maternal } \\
\text { Education }\end{array}$ & Formal Schooling & $508(80.1)$ & 1.00 \\
\cline { 2 - 5 } & Illiterate & $126(19.9)$ & 1.00 & $1.53(1.16-3.04)$ \\
\hline
\end{tabular}

Table 2: Independent correlates of Knowledge of STls symptoms among 3543 high school adolescents in Addis Ababa, Ethiopia, 2009.

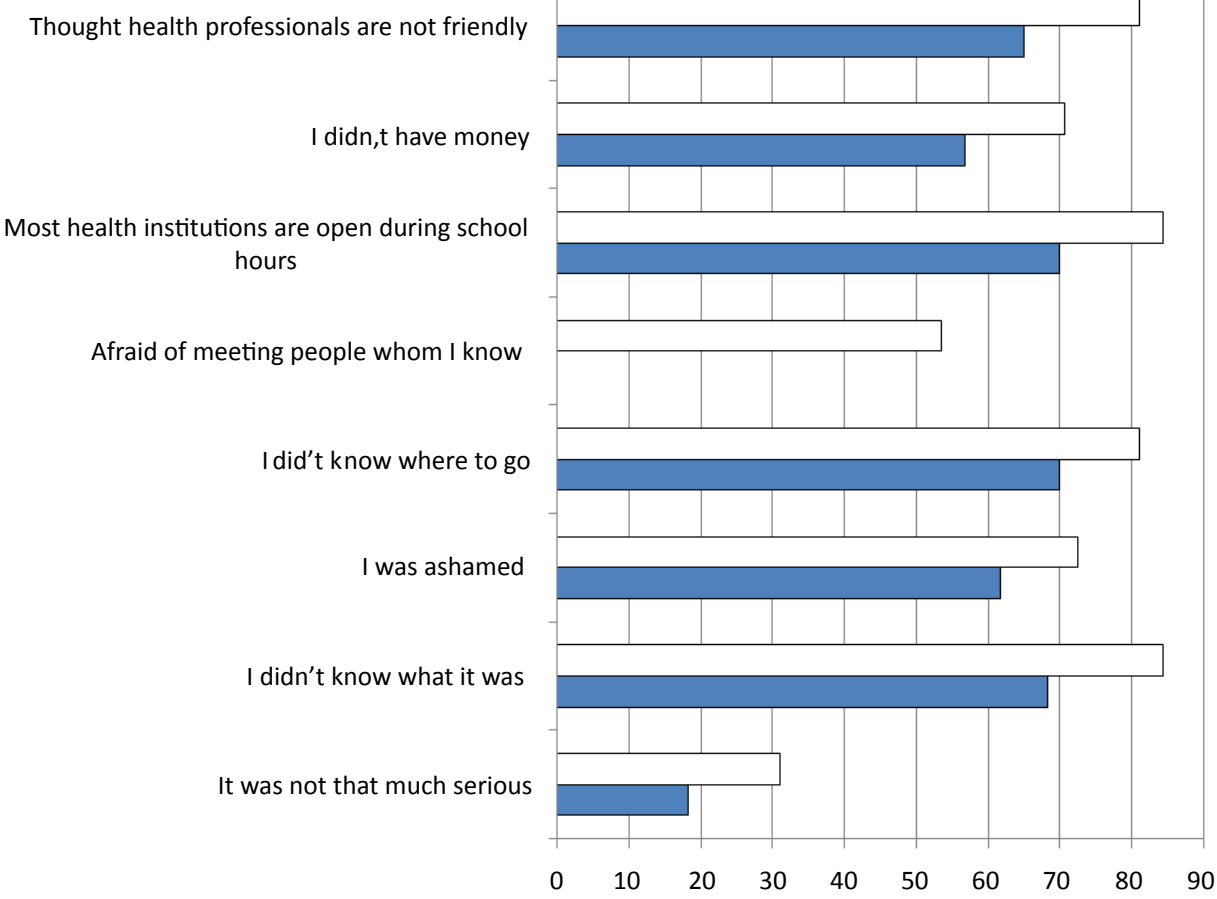

$\square$ Reason for not seeking treatment $\quad \square$ Reason for delayed treatment

Figure 1: Reasons for delayed or never sought treatment for STIs among High School Adolescents. 
Citation: Cherie A, Berhane Y (2012) Knowledge of Sexually Transmitted Infections and Barriers to Seeking Health Services among High School Adolescents in Addis Ababa, Ethiopia. J AIDS Clinic Res 3:153. doi:10.4172/2155-6113.1000153

Page 4 of 6

Unavailability of STIs services targeting adolescents: Discussants in almost all groups emphasized that the existing health institutions are not for adolescents it is either for adults or children, adolescent health is not given attention at all. Below are some of the sayings of the discussants regarding to this:

"The existing health services are not generally meant for adolescents. Most do not want to get treatment in health institutions for sexual related issues because the setting is not meant for adolescents it is either for small children or for adults. Thus, adolescents prefer to self threat their STI symptoms rather than going to health facilities."

Unfriendly and untrained health professionals on adolescents SRH and unresponsive health care system to adolescents need: Focus group participants in all male and female groups were complaining about the attitude and perceptions of health professionals, the medical training curriculum and the unresponsive health care system to adolescent health in general and STIs in particular. It was expressed as follows: "Adolescents do not have place in the health institutions. No service is targeted for adolescents and it is not surprising if health providers are not friendly to adolescents." I even doubt whether they [health care providers] have been taught about problems of adolescents during their formal training."

"It is apparent that the health needs of adolescents are ignored by the health care system. Once I went to one of the health institutions for a health problem. I was referred back and forth between adult and pediatric units because they both claimed serving only specific age categories... a friend of mine visited one of the health facilities in town for STI symptoms, he was very disappointed in the way he was treated by the health professionals... they were not friendly and respectful".

Lack of knowledge of where to seek treatment for STIs: Lack of knowledge of where to seek treatment and feeling of shame and stigmatization were one of the factor raised by the discussants.

"Adolescents often do not know which health facilities threat STIs. The problem is compounded as they also don't consult others, even their friends, for such kind of health problems because of fear of being stigmatized /labeled".

Accessibility of health services: The second major barrier raised in the FGDs was difficulty to access available health services. Most public health services are available during working hours requiring missing classes to get the service. In addition, the services are not readily available and the waiting time is often long. Discussants said: is long."

"Health services are not available nearby school and the waiting time

"Working hours of the health institutions is also a problem. Most work during school hours. You must miss the day's classes to get treatment."

Affordability of health services: The third barrier expressed was the perceived high cost of health services. The issues of cost were expressed by discussants in all groups as follows: "Adolescents do not have money to pay for health services, they cannot afford to pay."

Acceptability of the service: The fourth barrier was acceptability of the services because of perceptions of lack of privacy and confidentiality. "When you go to health facilities you are treated with adults there is no privacy... you may meet someone whom you know. Adolescents also fear that the information they are providing may not be kept confidential."

\section{Preferred sexual and reproductive health services delivery model}

Regarding the preferred sexual and reproductive health services delivery model, discussants reported that they prefer to receive services at school by youth friendly health workers that respect the rights of adolescents. Discussants across all group said "We prefer to get sexual/reproductive health services and other health related services in our school...there is no school health service in almost all schools. The Government should give high priority to the establishment of school health services."

Another group said, "We need health services designed and organized for adolescents. The service has to be friendly and appropriate for young people with due respect the rights of adolescents, which works at convenient hours for students, which is safe, and which considers adolescents as a human being."

\section{Discussion}

The study found that knowledge about STIs is generally very low among school adolescents. It was less than one in five students who were able to identify two symptoms of STIs. One third of those who had STIs symptom never went or received treatment, nearly half of those who sought treatment received the treatment late and almost half of those who reported had received treatment took self medication. Major barriers for seeking treatment for STIs were lack of knowledge of the disease and health institutions providing the care, feeling of shame, availability, accessibility, affordability and accessibility of the existing institutions.

Some limitations of this study worth considering in interpreting the results of this study are briefly discussed below. Although the self administered questionnaire was designed to minimize social desirability bias leading to under or over reporting of responses it is not an absolute remedy for this kind of bias. So, additional effort was made to find the truth using qualitative inquiry; which offered useful explanations and better insight about issues surrounding sexual reproductive health matters in schools. Thus, the researchers believe that the information obtained provides a fair account of sexual and reproductive health issues among high school adolescents.

In line with previous reports this study showed that adolescents are poorly informed about STIs [26-30]. The finding that showed male students had better knowledge than females has been reported previously [28]. The findings in this study showed also students who had educated mothers had better knowledge than their counterparts.

One in 20 students reported symptoms of STIs. This is slightly higher than the findings from Butajira [26]. The possible explanation for this could be our respondents are from urban areas. Previous studies indicated that adolescents in urban areas engage more in pre marital sex than their rural counterparts [1]. It was found in this study one third of those who had STIs symptoms never received treatment. This is comparable with the findings of the study made in Nigeria [30]. Nearly half of those who sought treatment received the treatment late and almost half of those who reported had received treatment took self medication. This corroborates with the findings from Peru [31]. This has implication for STIs transmission and prevention. Apart from developing complication, those who delayed treatment may continue to have sexual intercourse in the period of non treatment and transmit the infections to their partners especially if they are engaged in unprotected multiple partnerships. In addition, those who self treat may not take full treatment or the right treatment this may lead to drug resistance 
Citation: Cherie A, Berhane Y (2012) Knowledge of Sexually Transmitted Infections and Barriers to Seeking Health Services among High School Adolescents in Addis Ababa, Ethiopia. J AIDS Clinic Res 3:153. doi:10.4172/2155-6113.1000153

and further transmission of the infection. Thus, adolescents should be informed about the consequences of late or self treatment and health services should be within the reach of adolescents.

Respondents reported several barriers that hinder adolescents from seeking STIs treatment. Major among them were inaccessibility, unaffordable and unacceptable health service in the existing health institutions. This is in accord with extant literatures on adolescent reproductive health service utilization [30,32-35]. However, some of the barriers identified by the adolescents such as the absence of adolescent health services in general and STIs services in particular were unique to our study. This signifies to programme planners, policy and decision makers for reorienting and establishing a youth friendly health services.

Conceptually youth friendly health services are in agreement with adolescents' needs and the need to improve accessibility of the services is in accordance with previous studies $[11,36]$. However, students prefer to get SRH services in schools staffed by youth friendly professionals, targeting specifically adolescents and which respects the right of adolescents rather than youth corner in the health institutions. This calls for the action of decision makers and the government for considering school health service as an avenue for students to get SRH services.

In general, adolescents are not aware of the symptoms of STIs. Treatment for STI is ether sought late or never. Major barriers for not seeking treatment for STIs were perception of unavailability, inaccessibility, unaffordable and unacceptable of services provided by the existing health institutions, lack of knowledge of the disease and place of service delivery and feeling of shame. The study findings had policy and programmatic implications. Efforts should be intensified to provide adolescent friendly services. The Government and private schools needs to establish adolescent friendly school health services where these large groups of students are spending most of their time. So that, adolescents can be provided with accessible, acceptable, affordable, confidential and flexible services can be provided in a youth friendly manner. Inclusion of basic facts about STIs in sexuality education and the school curriculum to boost up their knowledge of STIs and there by reduce the transmission and increase the prevention of STIs. Involve adolescents in the planning, and decision making of their SRH services and programmes. Further longitudinal studies are recommended to study the STIs treatment seeking patterns of adolescents.

Key Message: adolescents are not aware of the symptoms of STI. Treatment for STI is ether sought late or never.

\section{References}

1. Dehne KL, Riedner G (2001) Sexually transmitted infections among adolescents: the need for adequate health services. Reprod Health Matters 9: $170-183$.

2. Lewis DA, Latif AS, Ndowa $F$ (2007) WHO global strategy for the prevention and control of sexually transmitted infections: time for action. Sex Transm Infect 83: 508-509.

3. WHO (2001) Global prevalence and Incidence of Selected Curable Sexually Transmitted Infections: Overview and Estimates, Geneva.

4. Eaton DK, Kann L, Kinchen S, Shanklin S, Ross J, et al. (2010) Youth risk behavior surveillance - United States, 2009. MMWR Surveill Summ. 59: 1-142.

5. Cherie A, Mitkie G, Ismail S, Berhane Y (2005) Perceived sufficiency and usefulness of IEC materials and methods related to HIVIAIDS among high school youth in Addis Ababa, Ethiopia. Afr J Reprod Health 9: 66-77.

6. Barnett T, Parkhurst J (2005) HIVIAIDS: sex, abstinence, and behavior change. Lancet Infect Dis 5: 590-593.

7. Murphy EM, Greene ME, Mihailovic A, Olupot-Olupot P (2006) Was the "ABC" Approach (abstinence, being faithful, using condoms) responsible for Uganda's Decline in HIV? PLoS Med 3: e379.
8. Lillie TL, Pulerwitz J, Curbow B (2009) Kenyan in-School Youths' level of understanding of abstinence, being faithful and consistent condom use terms: implications for HIV-prevention programs. J Health Commun 14: 276-292.

9. Bankole A, Singh S, Woog V, Wulf D (2004) Risk and Protection: Youth and HIVIAIDS in sub-Saharan Africa. New York: The Alan Guttmacher Institute.

10. Mmari KN, Magnani RJ (2003) Does making clinic-based reproductive health services more youth-friendly increase service use by adolescents? Evidence from Lusaka, Zambia. J Adolesc Health 33: 259-270.

11. Erulkar AS, Onoka CJ, Phiri A (2005) What is youth-friendly? Adolescents preferences for reproductive health services in Kenya and Zimbabwe. Afr Reprod Health 9: 51-58.

12. Centers for Disease Control and Prevention. (2008a). Sexually transmitted disease surveillance 2007. Retrieved July 20, 2009.

13. Centers for Disease Control and Prevention. (2008b). Youth risk surveillanceUnited States 2007. MMWR 57. Retrieved July 9, 2009.

14. Duncan EM, Tibaus G, Pelzer A, Mehari L, Peutherer, et al. (1995) Prevalence and significance of sexually transmitted diseases among Ethiopian women attending antenatal clinics in Addis Ababa. Ethiop J Health Dev 9: 31-40.

15. Fitaw Y, Worku A (2002) High-risk sexual behaviour and patterns of condom utilization of the Gondar College of Medical Sciences (GCMS) students, northwest Ethiopia. Ethiop J Health Dev 16: 335-338.

16. Ismail S, Bitsueamlak H (1997) High-risk sexual behaviours for STD/HIV pregnancies and contraception among high school students in a rural town north-western Ethiopia. Ethiop J Health Dev 11: 29-36.

17. Tesfaye F, Kassaye M, Kebede D (2000) Community-based survey of sexually transmitted disease syndromes in Adami-Tullu. Ethiop J Health Dev 14: 7-12.

18. Taffa N, Bjune G, Sundby J, Gaustad P, Alestrøm A (2002) Prevalence of gonococcal and chlamydial infections and sexual risk behaviours among youth in Addis Ababa, Ethiopia. Sex Transm Dis 29: 823-833.

19. Matteelli A, Kassa A, Gerbase A, Farina C, Ghatel G, et al. (2000) Passive Sentinel Surveillance System for Sexually transmitted diseases in Primary healthcare sites in Ethiopia. Sex Transm Infect 76: 131-133.

20. Assefa A, Ishak A, Stevens R, Fergussen E, Giles M, et al. (1998) Prevalence of HIV, syphilis and genital chlamydial infection among women in North-West Ethiopia. Epidemiol Infect 120: 171-177

21. Federal Minstery of Health (2001) National guidelines for the management of sexually transmitted infections using the Syndromic Approach.

22. Ministry of Health of Ethiopia (2010) Health Sector Development Programme IV.

23. Cherie A, Berhane Y (2012) Oral and Anal Sex Practices among high School Youth in Addis Ababa, Ethiopia. BMC Public Health 12: 5.

24. Eaton DK, Kann L, Kinchen S, Shanklin S, Ross J, et al. (2010) Youth risk behavior surveillance - United States, 2009. MMWR Surveill Summ 59: 1-142.

25. DiClemente Ralph J, Salazar L, Crosby R (2007) A review of STD/HIV preventive interventions for adolescents: sustaining effects using an ecological approach. J Pediatr Psychol 32: 888-906.

26. Molla M, Emmelin M, Berhane Y, Lindtjørn B (2008) Readiness of youth in rural Ethiopia to seek health services for sexually transmitted infections AJAR 8: 135-146.

27. Trani F, Gnisci F, Nobile CG, Angelillo IF (2005) Adolescents and sexually transmitted infections: Knowledge and behaviour in Italy. J Paediatr Child Health 41: 260-264

28. Garside R, Ayres R, Owen M, Pearson VAH, Roizen J (2001) They neve tell you about the consequences: young people's awareness of sexually transmitted infections. Int J STD AIDS 12: 582-588.

29. Nzioka C (2001) Perspectives of adolescent boys on the risks of unwanted pregnancy and sexually transmitted infections: Kenya. Reprod Health Matters 9: 108-117.

30. Mmari KN, Oseni O, Fatusi AO (2010) STIs Treatment Seeking Behaviours among youth in Nigeria: Are there gender differences? Int Perspect Sex Reprod Health 36: 72-79. 
Citation: Cherie A, Berhane Y (2012) Knowledge of Sexually Transmitted Infections and Barriers to Seeking Health Services among High School Adolescents in Addis Ababa, Ethiopia. J AIDS Clinic Res 3:153. doi:10.4172/2155-6113.1000153

Page 6 of 6

31. Garcia PJ, Chavez S, Feringa B, Chiappe M, Li W, et al. (2004) Reproductive tract infections in rural women from the highlands, jungle, and coastal regions of Peru. Bull World Health Organ 82: 483-492.

32. WHO (2001) Global consultation on adolescent health services a consensus statement. Geneva, Department of Child and Adolescent Health and Development, World Health Organization.

33. Agampodi SB, Agampodi TC, Ukd P (2008) Adolescents perception of reproductive health care services in SriLanka. BMC Health Serv Res 8: 98.
34. Katz K, Naré C (2002) Reproductive health knowledge and use of services among young adults in Dakar, Senegal. J Biosoc Sci 34: 215-231.

35. Warenius LU, Faxelid EA, Chishimba PN, Musandu JO, Ong'any AA et al (2006) Nurse-midwives' attitudes towards adolescent sexual and reproductive health needs in Kenya and Zambia. Reprod Health Matters 14: 119-128.

36. WHO (2006) Steady, Ready, Go. Geneva, World Health Organization. 